\title{
TÄCKLIND UNIQUENESS CLASSES FOR HEAT EQUATION ON NONCOMPACT RIEMANNIAN MANIFOLDS
}

\author{
V.F. VIL'DANOVA, F.KH. MUKMINOV
}

\begin{abstract}
We describe uniqueness classes for solution of the Cauchy problem for the heat equation on a connected noncompact complete Riemannian manifold. For the case of manifolds with boundary, we assume that the solution satisfies the Dirichlet and Neumann conditions on the boundary.

Uniqueness classes are determined by a non-negative function growing no faster than the distance from a fixed point along a geodesics. The classes are similar to uniqueness classes of Täcklind type for the equation on the real line.
\end{abstract}

Keywords:uniqueness classes, heat equation, Riemannian manifold.

Mathematics Subject Classification: 35K10, 35K20, 35R01, 58J32

\section{INTRODUCTION}

Let $M$ be a geodesically complete non-compact connected Riemannian manifold of dimension $n$. Let $\triangle$ be the Laplace operator (or, the same, Laplace-Beltrami operator) on $M$. As it is known, in local coordinates $x_{1}, x_{2}, \ldots, x_{n}$, Laplacian $\triangle$ reads as

$$
\triangle=\frac{1}{\sqrt{g}} \sum_{i, j=1}^{n} \frac{\partial}{\partial x_{i}}\left(\sqrt{g} g^{i j} \frac{\partial}{\partial x_{j}}\right),
$$

where $g^{i j}$ are contravariant components of the metric tensor (in distinction to covariant component $\left.g_{i j}\right), g=\operatorname{det}\left\|g_{i j}\right\|$. In the cylindrical domain $D^{T}=(0, T) \times M$, we consider the heat equation:

$$
u_{t}-\triangle u=0 .
$$

The work is devoted to the proof of the uniqueness of the solution to Cauchy problem for equation (1) in unbounded domain $D^{T}$. A fundamental work is that by A.N. Tikhonov [1], where not only the uniqueness class $|u(t, x)| \leqslant B \exp \left(b|x|^{2}\right)$ was provided for the heat equation on the real axis, but also there was constructed a non-uniqueness example for the Cauchy problem. In work [2], S. Täklind specified this result by showing that the uniqueness of the

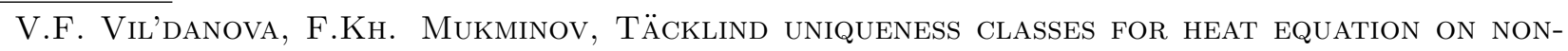
COMPACT

RIEMANNIAN MANIFOLDS.

(c) Vil'danova V.F., Mukminov F.KH., 2014.

The work is supported by RFBR (grant no. 13-01-00081-a).

Submitted November 24, 2014. 
solution to the Cauchy problem holds true in the class of functions $|u(t, x)| \leqslant B \exp (|x| h(|x|))$, where $h(r)$ is a non-decreasing non-negative function with the diverging integral

$$
\int_{1}^{\infty} \frac{d r}{h(r)}=+\infty
$$

In what follows, such functions $h$ are called Täcklind functions. At that, for each function with the diverging integral $\int_{1}^{\infty} \frac{d r}{h_{1}(r)}<\infty$, there was constructed an example of non-zero solution to the Cauchy problem for the one-dimensional heat equation satisfying the estimate $|u(t, x)| \leqslant$ $B \exp \left(|x| h_{1}(|x|)\right)$ and the condition $u(0, x)=0$.

These results were generalized for a general parabolic equation in $\mathbb{R}^{n}$ in works [3] - [16] and others. A detailed survey of these works was provided, for instance, in [3]. It was mentioned in [4] and [5] that in the case of mixed problem, an adequate expression of a uniqueness class is that in terms of growth of the integrals

$$
\int_{0}^{T} \int_{\{|x|<r\} \cap M} u^{2}(t, x) d x d t \leqslant B \exp (r h(r)) .
$$

Anisotropic uniqueness classes were considered in works [6], [7. In the case of the mixed Dirichlet problem there were found geometric uniqueness classes being wider that the Täcklind ones if domain $M \subset \mathbb{R}^{n}$ converges quite fast at infinity (see [3]). The dependence of the uniqueness classes of lower order coefficients was studied in work 8 .

We impose the Dirichlet and Neumann conditions on the lateral boundary of cylinder $D^{T}$ :

$$
\left.u(t, x)\right|_{\Gamma_{1}}=0 ;\left.\quad \frac{\partial u}{\partial N}\right|_{\Gamma_{2}}=0 .
$$

Here $\gamma \subset \partial M$ is an arbitrary closed subset, $\Gamma_{1}=(0, T) \times \gamma$ and $\Gamma_{2}$ is its complement: $\Gamma_{2}=\Gamma \backslash \Gamma_{1}$. The initial function

$$
u(0, x)=\varphi(x) \in L_{2, l c}(M)
$$

is assumed to be square integrable over each bounded subset $Q \subset M$.

Let $\sigma(x), x \in M$, be a positive locally Lipschitz function having bounded level surfaces, $|\nabla \sigma(x)| \leqslant 1, \sigma(x) \rightarrow \infty$ as $\operatorname{dist}\left(x, x_{0}\right) \rightarrow \infty$.

We let $M(r)=\{x \in M \mid \sigma(x)<r\}, D_{\rho}^{T, r}=(0, T) \times(M(r) \backslash M(\rho))$, (index $\rho=0$ will be omitted).

Theorem 1. Let $u(t, x)$ be the solution in $D^{T}$ to problem (1), (3) with the initial condition

$$
u(0, x)=0
$$

If there exists a Täcklind function $h(r)$ such that for each $r \geqslant 1$

$$
\|u\|_{D^{T, r}}^{2} \leqslant \exp (r h(r))
$$

then $u(t, x) \equiv 0$ in $D^{T}$. 


\section{Generalized SOlution to Problem}

Let $D_{a}^{b}=(a, b) \times M, D^{T}=D_{0}^{T}$. We introduce the following notations in local charts:

$$
\langle\bar{u}, \bar{v}\rangle_{g}=\sum_{i, j=1}^{n} g_{i j} u^{i} v^{j}, \quad(\nabla f)^{i}=\sum_{j=1}^{n} g^{i j} \frac{\partial f}{\partial x_{j}} .
$$

It is easy to see that

$$
\langle\nabla f, \nabla w\rangle_{g}=\sum_{i, j=1}^{n} g^{i j} \frac{\partial f}{\partial x_{i}} \frac{\partial w}{\partial x_{j}}, \quad|\nabla f|^{2}=\langle\nabla f, \nabla f\rangle_{g} .
$$

The measure on the Riemannian manifold will be denoted by $d \nu$.

We introduce the scalar product

$$
(u, w)_{H^{0,1}}=\int_{D^{T}}\left(u w+\langle\nabla u, \nabla w\rangle_{g}\right) d \nu d t .
$$

on the set of compactly supported functions in $C^{1}\left(\overline{D^{T}}\right)$. By $C_{0}^{1}\left(D^{T} \backslash \Gamma_{1}\right)$ we denote the set of functions in $C^{1}\left(\overline{D^{T}}\right)$ vanishing in the vicinity of $\Gamma_{1}$ and having a compact support. We denote the completion of this linear space w.r.t. the introduced scalar product by $\stackrel{\circ}{H}^{0,1}\left(D^{T} ; \Gamma_{1}\right)$.

A generalized solution to problem (1), (3), (4) in $D^{T}$ is a function $u(t, x)$ such that for each compactly supported Lipschitz function $\eta(x)$ the product $u \eta \in \stackrel{\circ}{H}^{0,1}\left(D^{T} ; \Gamma_{1}\right)$ satisfies the integral identity

$$
\int_{D^{T}}\left(-u v_{t}+\langle\nabla u, \nabla v\rangle_{g}\right) d \nu d t=\int_{M} \varphi(x) v(0, x) d \nu,
$$

where $v(t, x) \in C_{0}^{1}\left(D^{T} \backslash \Gamma_{1}\right)$ is an arbitrary function such that $v(T, x)=0$.

Let us show that we can apply the standard technique of Steklov's averaging (see, for instance, [18, Ch. 3, Sect. 2])

$$
u_{h}(t, x)=\frac{1}{h} \int_{0}^{h} u(t+\tau, x) d \tau
$$

to the generalized solution of problem (1), (3), (4). First we observe the boundedness of the shift operators $T_{z} v(t)=v(t+z)$ and Steklov's averaging operators in space $\stackrel{\circ}{H}^{0,1}\left(D^{T} ; \Gamma_{1}\right)$ under the assumptions that function $v(t)$ is continued by zero for $t \leqslant 0$ and $t \geqslant T-h$ :

$$
\begin{aligned}
\left\|T_{z} v\right\|_{H^{0,1}\left(D^{T}\right)} & \leqslant\|v\|_{H^{0,1}\left(D^{T}\right)}, \\
\left\|v_{h}\right\|_{H^{0,1}\left(D^{T}\right)} & \leqslant\|v\|_{H^{0,1}\left(D^{T}\right)} .
\end{aligned}
$$

Let us establish first an integral relation required for future calculations. Replace function $v$ by $v_{-h}, v \in C_{0}^{1}\left(D^{T} \backslash \Gamma_{1}\right)$ in $(7)$, it is easy to get the identity

$$
\int_{D^{T}}\left(\left(u_{h}\right)_{t} v+\left\langle(\nabla u)_{h}, \nabla v\right\rangle_{g}\right) d \nu d t=0
$$

which is also valid for functions $v(t, x) \in V$, where $V$ is formed by the elements of space $\stackrel{\circ}{H}^{0,1}\left(D^{T} ; \Gamma_{1}\right)$ vanishing as $t>T-\delta, \delta>0$ and having a compact support. In particular, if 
$\eta(x)$ is a Lipschitz function with a bounded support, substituting $v=\eta(x) u_{h}^{\tau}$, where $\left(u_{h}\right)^{\tau}$ is function $u_{h}$ continued by zero for $t>\tau$, we obtain

$$
\int_{D^{\tau}}\left[\frac{1}{2}\left(\left(u_{h}^{\tau}\right)^{2} \eta\right)_{t}+\left\langle(\nabla u)_{h}, \nabla\left(\eta u_{h}^{\tau}\right)\right\rangle_{g}\right] d \nu d t=0 .
$$

Let us justify the possibility of passing to the limit as $h \rightarrow 0$ in identity (10) to the relation

$$
\int_{M}\left(u^{2}(\tau, x)-\varphi^{2}(x)\right) \eta d \nu+2 \int_{D^{\tau}}\langle\nabla u, \nabla(\eta u)\rangle_{g} d \nu d t=0 .
$$

In such passage, the compactness of the support of function $\eta$ allows us to assume that function $u \eta$ is an element of space $\stackrel{\circ}{H}^{0,1}\left(D^{T} ; \Gamma_{1}\right)$. For instance, let us show that

$$
\int_{D^{T}}\left\langle(\nabla u)_{h}, \nabla\left(v_{h}\right)\right\rangle_{g} d \nu d t \rightarrow \int_{D^{T}}\langle\nabla u, \nabla v\rangle_{g} d \nu d t
$$

as $h \rightarrow 0$ and $v \in V$. In order to do it, we consider the expression

$$
\begin{aligned}
& \left|\int_{D^{T}}\left\langle\nabla u, \nabla\left(v_{h}\right)_{-h}-\nabla v_{h}\right\rangle_{g} d \nu d t\right|=\left|\int_{D^{T}}\left\langle\frac{\nabla u}{h}, \int_{-h}^{0} \nabla\left(v_{h}(t+z)-v_{h}(t)\right) d z\right\rangle_{g} d \nu d t\right| \\
& \leqslant \int_{-h}^{0} \frac{d z}{h} \int_{D^{T}}\left\langle\left(\nabla u, \nabla\left(v_{h}(t+z)-v_{h}(t)\right)\right)\right\rangle_{g} d \nu d t \leqslant \int_{-h}^{0} \frac{d z}{h}\|u \eta\|_{H^{0,1}}\left\|T_{z} v_{h}-v_{h}\right\|_{H^{0,1}} \equiv I,
\end{aligned}
$$

where a compactly supported Lipschitz function $\eta$ is equal to 1 in the vicinity of the support of function $v$. We choose an arbitrary $\varepsilon>0$. Employing the equicontinuity of the family of functions $v_{h}$ and $\nabla v_{h}$ and choosing a sufficiently small $h$, as $|z| \leqslant h$, we arrive at the estimate

$$
\left\|T_{z} v_{h}-v_{h}\right\|_{H^{0,1}\left(D^{T}\right)} \leqslant \varepsilon
$$

for function $v \in C_{0}^{1}\left(D_{0}^{T-\delta}\right)$. It implies the inequality $I \leqslant C \varepsilon$. Thanks to the boundedness of the shift operators and Steklov's averaging operator, the latter estimate is also true fo functions $v(t, x) \in V$. Exactly in the same way we can establish the smallness of the quantity

$$
\left|\int_{D^{T}}\left\langle\nabla u, \nabla v_{h}-\nabla v\right\rangle_{g} d \nu d t\right| \leqslant C \varepsilon
$$

for sufficiently small $h$.

Thus, we have justified passage to the limit (12). In particular, letting $v_{h}=\eta(x) u_{h}^{\tau}(t, x)$ in 12 . we obtain that

$$
\int_{D^{T}}\left\langle(\nabla u)_{h}, \nabla\left(\eta u_{h}^{\tau}\right)\right\rangle_{g} d \nu d t \rightarrow \int_{D^{T}}\left\langle\nabla u, \nabla\left(\eta u^{\tau}\right)\right\rangle_{g} d \nu d t, \quad h \rightarrow 0 .
$$

Employing (14), we pass to the limit and come to (11) from (10). Of course, at that we employ the belonging $u(t, x) \eta(x) \in C\left([0, T] ; L_{2}(M)\right)$, which can be proven by (9) in a standard way (see, for instance, [18, Ch. 3, Sect. 4, Lm. 4.1]). 


\section{Proof of Theorem 1}

We follow the technique of work [5]. The theorem will follow the next statement.

Proposition 1. Let $\varphi(x)=0, x \in M(R)$, and $u(t, x)$ be a solution to problem (1), (3), (4). Then for each $t>0, r \in(0, R]$, the inequality

$$
H^{t, r}(u) \leqslant \exp \left(1-2 \kappa_{1} t^{-1}(R-r)^{2}\right) \max _{\tau \in[0, t]} H^{\tau, R}(u)
$$

holds true, where

$$
H^{t, r}(u)=\int_{M(r)} u^{2}(t, x) d \nu
$$

$\kappa_{1}=1 /\left(16 e^{2}\right)$.

Proof. Let $r \leqslant R, \xi(\tau, r, \rho)$ be a continuous non-negative function equalling one as $\tau \leqslant r-\rho$ and vanishing as $\tau \geqslant r$. In the remaining interval it satisfies the condition $\frac{\partial \xi}{\partial \tau}=-1 / \rho$. We substitute $\eta(x)=\xi^{2}(\sigma(x), r, \rho)$ in identity (9). Then by the condition $\eta \varphi \equiv 0$ and (11), one can get easily the inequalities

$$
\begin{aligned}
\int_{M} \eta u^{2}(T, x) d \nu & +2 \int_{D^{T}}\langle\eta \nabla u, \nabla u\rangle_{g} d \nu d t \\
& \leqslant 2 \int_{D^{T}}\left|\langle\nabla u, u \nabla \eta\rangle_{g}\right| d \nu d t \leqslant 2 \int_{D^{T}}\left(\varepsilon \xi^{2}|\nabla u|^{2}+\varepsilon^{-1} u^{2}|\nabla \xi|^{2}\right) d \nu d t
\end{aligned}
$$

for each $r>\rho>0$. Transforming the latter as $\varepsilon=1 / 2$, we get

$$
\int_{M} \xi^{2} u^{2}(T, x) d \nu \leqslant 4 \int_{D^{T}} u^{2}|\nabla \xi|^{2} d \nu d t .
$$

Employing the definition of function $\xi$, it is easy to obtain the inequality

$$
\int_{M(r-\rho)} u^{2}(t, x) d \nu \leqslant \frac{4}{\rho^{2}} \int_{0}^{t} \int_{M(r) \backslash M(r-\rho)} u^{2} d \nu d t .
$$

We write the latter in terms of function $H$ :

$$
H^{t, r-\rho}(u) \leqslant \frac{4}{\rho^{2}} \int_{0}^{t} H^{\tau, r}(u) d \tau .
$$

We observe that choosing $\varepsilon=1 / 4$, one can also get the inequality

$$
\int_{M(r-\rho)} u^{2}(t, x) d \nu+\frac{1}{2} \int_{D^{T, r-\rho}}|\nabla u|^{2} d \nu d t \leqslant \frac{8}{\rho^{2}} \int_{0}^{t} \int_{M(r) \backslash M(r-\rho)} u^{2} d \nu d t .
$$

We shall apply inequality (16) inductively for sequence $r_{i}=R-k \rho, i=0,1,2, \ldots k$, where $\rho=(R-r) / k$. Number $k$ will be fixed later. Taking into account that $H^{t, r}(u) \leqslant A=$ $\max _{\tau \in[0, t]} H^{\tau, R}(u)$, we have

$$
H^{t, r_{1}}(u)=H^{t, r_{0}-\rho}(u) \leqslant \frac{4 A t}{\rho^{2}}
$$


By induction in $k$ we establish the inequality

$$
H^{t, r_{k}}(u) \leqslant \frac{A 4^{k} t^{k}}{\rho^{2 k} k !}
$$

Employing Stirling's inequality, by (18) one can get easily

$$
H^{t, r_{k}}(u) \leqslant \frac{A 4^{k} e^{k} t^{k}}{\sqrt{2 \pi k} \rho^{2 k} k^{k}} \leqslant A e^{-k \ln \frac{\rho^{2} k}{4 e t}} .
$$

We choose number $k$ so that $4 e^{2} t \leqslant \rho^{2} k \leqslant 8 e^{2} t$. Then it follows from 19 that $H^{t, r_{k}}(u) \leqslant A e^{-k}$. Then, $(R-r)^{2}=\rho^{2} k^{2} \leqslant 8 e^{2} t k$. As $k$, we choose the smallest natural number obeying this inequality. Then $k \geqslant \frac{(R-r)^{2}}{8 e^{2} t}, \rho^{2} k^{2}>8 e^{2} t(k-1)$, and $\rho^{2} k>4 e^{2} t$ as $k \geqslant 2$. Thus, inequality (15) is established for $k \geqslant 2$. As $k=1$, it is trivial.

Corollary. Let $u(t, x)$ be the generalized solution to problem (1), (3), (4) in $D^{T}, T \leqslant 1$. Then for each $t \in(0, T], r \in[0, R)$, the identity

$$
H^{t, r}(u) \leqslant 12 \exp \left(-2 \kappa_{1} t^{-1}(R-r)^{2}\right) \max _{\tau \in[0, t]} H^{\tau, R}(u)+14\|\varphi\|_{M(R)}^{2}
$$

holds true.

Proof. Let $v(t, x)$ be the solution to problem (1), (3), (4) with the initial function $\varphi(x) \chi_{M(R)} \in$ $L_{2}(M)$, where $\chi_{M(R)}$ is the characteristic function of set $M(R)$. By (11) with $\eta=1$, it is easy to prove the inequality

$$
\|v(t)\|^{2}+\int_{0}^{t} \int_{M}|\nabla v|^{2} d \nu d t \leqslant\|\varphi\|_{M(R)}^{2}
$$

for $v(t, x)$. It follows that

$$
\|\varphi\|_{M(R)}^{2} \geqslant \max _{\tau \in[0, t]} H^{\tau, R}(v), \quad t \in(0, T] .
$$

Function $w(t, x)=u(t, x)-v(t, x)$ satisfies the assumption of Proposition 1, therefore, for $t \in(0, T], r \in[0, R)$ we have

$$
\begin{aligned}
H^{t, r}(u) & \leqslant 2 H^{t, r}(w)+2 H^{t, r}(v) \\
& \leqslant 4 \exp \left(1-2 \kappa_{1} t^{-1}(R-r)^{2}\right)\left\{\max _{\tau \in[0, t]} H^{\tau, R}(u)+\max _{\tau \in[0, t]} H^{\tau, R}(v)\right\}+2 H^{t, R}(v) .
\end{aligned}
$$

Employing (22), by the latter relation we arrive at inequality (20).

Proof of Theorem 1. We fix arbitrary $t \in(0, T]$. We take arbitary $R_{0}>1$ and estimate $\|u(t)\|_{M\left(R_{0}\right)}^{2}$ as follows. Consider the sequence $R_{k}=2^{k} R_{0}$ and let

$$
\Delta t_{k}=\frac{\kappa_{1}}{4} \frac{R_{k}}{h\left(R_{k}\right)}, \quad k=1,2, \ldots
$$

By the monotonicity of function $h(r)$ we have

$$
\sum_{k=1}^{p} \Delta t_{k}=\frac{\kappa_{1}}{4} \sum_{k=1}^{p} \frac{R_{k+1}-R_{k}}{h\left(R_{k}\right)} \geqslant \frac{\kappa_{1}}{4} \int_{R_{1}}^{R_{p+1}} \frac{d r}{h(r)} \rightarrow \infty \quad \text { as } \quad p \rightarrow \infty .
$$

Therefore, for each choice of number $R_{0}$ there exists a number $p$ such that

$$
\sum_{k=1}^{p+1} \Delta t_{k} \geqslant t
$$


Let $p=p\left(R_{0}\right)$ the smallest among indices $p$ satisfying inequality 24 so that $\sum_{k=1}^{p} \Delta t_{k}<t$. We redefine $\Delta t_{p+1}$ by the identity

$$
\Delta t_{p+1}=t-\sum_{k=1}^{p} \Delta t_{k} \leqslant \frac{\kappa_{1}}{4} \frac{R_{p+1}}{h\left(R_{p+1}\right)} .
$$

We introduce a decreasing sequence of times $t_{0}=t, t_{1}=t_{0}-\Delta t_{1}, t_{2}=t_{1}-\Delta t_{2}, \ldots, t_{p+1}=$ $t_{p}-\Delta t_{p+1}=0$. In view of 23), 25) we obviously have inequalities

$$
\frac{\frac{\kappa_{1}}{2} R_{k}^{2}}{\Delta t_{k}} \geqslant 2 R_{k} h\left(R_{k}\right), \quad k=\overline{1, p+1}
$$

By $(16)$ and $(6)$ for $k \geqslant 1$ we get the relations

$$
\|u(t)\|_{M\left(R_{k-1}\right)}^{2} \leqslant S_{k} \int_{0}^{T}\|u(t)\|_{M\left(R_{k}\right)}^{2} d t \leqslant S_{k} \exp \left(R_{k} h\left(R_{k}\right)\right), \quad t \in(0, T],
$$

where

$$
S_{k}=4\left(R_{k}-R_{k-1}\right)^{-2}=16\left(R_{k}\right)^{-2} \leqslant 16\left(R_{1}\right)^{-2} \equiv S / 12 .
$$

Treating function $u(t, x)$ as the solution to the problem for equation (1) with initial conditions at $t=t_{1}$, by means of inequality $(20)$ and the definition of sequence $R_{k}$ we obtain the relation

$$
\left\|u\left(t_{0}\right)\right\|_{M\left(R_{0}\right)}^{2} \leqslant 12 \exp \left(-2 \kappa_{1} \frac{\left(\frac{R_{1}}{2}\right)^{2}}{\triangle t_{1}}\right) \max _{t \in\left[t_{1}, t_{0}\right]}\|u(t)\|_{M\left(R_{1}\right)}^{2}+14\left\|u\left(t_{1}\right)\right\|_{M\left(R_{1}\right)}^{2} .
$$

We employ inequalities (27), 26):

$$
\begin{aligned}
\left\|u\left(t_{0}\right)\right\|_{M\left(R_{0}\right)}^{2} & \left.\leqslant S \exp \left(R_{1} h\left(R_{1}\right)\right)-\kappa_{1} \frac{R_{1}^{2}}{2 \triangle t_{1}}\right)+14\left\|u\left(t_{1}\right)\right\|_{M\left(R_{1}\right)}^{2} \\
& \leqslant S \exp \left(-R_{1} h\left(R_{1}\right)\right)+14\left\|u\left(t_{1}\right)\right\|_{M\left(R_{1}\right)}^{2} .
\end{aligned}
$$

Completely in the same way one can prove the inequalities as $k=\overline{2, p}$ :

$$
\begin{aligned}
\left\|u\left(t_{1}\right)\right\|_{M\left(R_{1}\right)}^{2} & \left.\leqslant S \exp \left(R_{2} h\left(R_{2}\right)\right)-\kappa_{1} \frac{R_{2}^{2}}{2 \triangle t_{2}}\right)+14\left\|u\left(t_{2}\right)\right\|_{M\left(R_{2}\right)}^{2} \\
& \leqslant S \exp \left(-R_{2} h\left(R_{2}\right)\right)+14\left\|u\left(t_{2}\right)\right\|_{M\left(R_{2}\right)}^{2}, \\
\left\|u\left(t_{k-1}\right)\right\|_{M\left(R_{k-1}\right)}^{2} & \leqslant S \exp \left(-R_{k} h\left(R_{k}\right)\right)+14\left\|u\left(t_{k}\right)\right\|_{M\left(R_{k}\right)}^{2} .
\end{aligned}
$$

Since $t_{p+1}=0$, by applying (27), (26) we find that

$$
\left.\left\|u\left(t_{p}\right)\right\|_{M\left(R_{p}\right)}^{2} \leqslant S \exp \left(-R_{p+1} h\left(R_{p+1}\right)\right)\right) .
$$

Multiplying $(29), k=\overline{1, p+1}$, by $14^{k-1}$ and summing up, for $R_{0} \geqslant 1$ we obtain

$$
\begin{aligned}
\|u(t)\|_{M\left(R_{0}\right)}^{2} & \leqslant S\left\{\exp \left(-R_{1} h\left(R_{1}\right)\right)+\ldots+14^{p} \exp \left(-R_{p+1} h\left(R_{p+1}\right)\right)\right\} \\
& \leqslant S\left\{\exp \left(-R_{1} h\left(R_{1}\right)\right) \ldots+14^{p} \exp \left(-2^{p} R_{1} h\left(R_{1}\right)\right)\right\} \leqslant C_{1}\left(R_{1}\right)^{-2} .
\end{aligned}
$$

Thus, we have established a non-negative monotonically non-decreasing function $\|u(t)\|_{M\left(R_{0}\right)}^{2}$ of $R_{0}$ tends to zero as $R_{0} \rightarrow \infty$. Therefore, $\|u(t)\|_{M\left(R_{0}\right)}^{2}=0$ for each $R_{0}>0$. Since $t \in(0, T]$ is arbitrary, solution $u(t, x)$ vanishes identically in $D^{T}$. 


\section{UNIQUENESS CLASSES AND EXAMPLES}

As the simplest example, we consider the manifold $M=\mathbb{R}^{n}$ with the metric $g_{i j}=(\rho(|x|))^{-1} \delta_{i j}$, where $\delta_{i j}$ is the Kronecker delta, $\rho$ is a positive continuous function. Heat equation (1) becomes

$$
u_{t}=\rho^{\frac{n}{2}}(|x|) \sum_{i=1}^{n}\left(\rho^{-\frac{n}{2}}(|x|) u_{x_{i}}\right)_{x_{i}} .
$$

Let $\sigma=\sigma(|x|)$. We choose function $\sigma$ so that $|\nabla \sigma|^{2}=1$,

$$
|\nabla \sigma|^{2}=\sum_{i, j=1}^{n} g^{i j} \sigma_{x_{i}} \sigma_{x_{j}}=\sum_{i=1}^{n} g^{i j} \rho \sigma_{x_{i}}^{2}=\rho\left(\sigma^{\prime}\right)^{2}=1 .
$$

Then $\sigma(s)=\int_{0}^{s} \frac{d r}{\sqrt{\rho(r)}}$. Since $\sigma(|x|)<r \Leftrightarrow|x|<\sigma^{-1}(r)=s$, then $D^{T, r}=\left\{(t, x) \in D^{T}|| x \mid<s\right\} \equiv D^{T}(s)$. Therefore, condition 6 is written as

$$
\int_{D^{T}(s)} u^{2}(t, x) \rho^{-\frac{n}{2}}(|x|) d x d t \leqslant \exp (2 \sigma(s) h(\sigma(s))) .
$$

Let $M=\mathbb{R}^{2}$ with the metric $g_{i j}=\left(\rho_{i}\left(x_{i}\right)\right)^{-1} \delta_{i j}$. We choose function $\sigma(x)=\sqrt{f\left(x_{1}\right)+v\left(x_{2}\right)}$, where $f(r)=\left(\int_{0}^{r} \frac{d x_{1}}{\sqrt{\rho_{1}\left(x_{1}\right)}}\right)^{2}$ and $v(r)=\left(\int_{0}^{r} \frac{d x_{1}}{\sqrt{\rho_{2}\left(x_{1}\right)}}\right)^{2}$. Then $|\nabla \sigma|^{2}=\sum_{i=1}^{2} \rho_{i} \sigma_{x_{i}}^{2}=1$. Heat equation (1) reads as

$$
u_{t}=\sqrt{\rho_{1}\left(x_{1}\right) \rho_{2}\left(x_{2}\right)} \sum_{i=1}^{2}\left(\frac{1}{\sqrt{\rho_{1}\left(x_{1}\right) \rho_{2}\left(x_{2}\right)}} u_{x_{i}}\right)_{x_{i}},
$$

and condition (6) is written as

$$
\int_{D^{T, r}} \frac{u^{2}(t, x)}{\sqrt{\rho_{1}\left(x_{1}\right) \rho_{2}\left(x_{2}\right)}} d x d t \leqslant \exp (2(r h(r)) .
$$

\section{BIBLIOGRAPHY}

1. A.N. Tikhonov. Théorèmes d'unicité pour l'équation de la chaleur // Matem. Sborn. 42:2, 199-216 (1935). (in French.)

2. S. Täcklind. Sur les class quasianalytiques des solutions des équations aux deriveés partielles du type parabolique // Nova Acta Reg. Soc. Schi. Uppsal. Ser. 10:3, 3-55 (1936).

3. L.M. Kozhevnikova. Uniqueness classes for solutions in unbounded domains of the first mixed problem for the equation $u_{t}=A u$ with quasi-elliptic operator A // Matem. Sborn. 198:7, 59-102 (2007). [Sb. Math. 198:7, 55-96 (2007).]

4. O.A. Oleinik, E.V. Radkevich. The method of introducing a parameter in the study of evolutionary equations // Uspekhi Matem. Nauk. 33:5, 7-76 (1978). [Russ. Math. Surv. 33:5, 7-84 (1978).]

5. A.K. Gushchin. On the uniform stabilization of solutions of the second mixed problem for a parabolic equation // Matem. Sborn. 119(161):4(12), 451-508 (1982). [Math. USSR Sb. 47:2, 439-498 (1984).]

6. I.M. Sonin. On uniqueness classes for degenerating parabolic equations // Matem. Sborn. 85(127):4(8), 459-473 (1971). [Math. USSR Sb. 14:4, 453-469 (1971).]

7. L.I. Kamynin. The uniqueness of the solution of the first boundary value problem in an unbounded domain for a second-order parabolic equation // Zhurn. Vychisl. Matem. Matem. Fiz. 24:9, 13311345 (1984). [USSR Comp. Math. Math. Phys. 24:5, 32-40 (1984).] 
8. J.I. Žitomirskil. Uniqueness classes for solutions of the Cauchy problem for linear equations with rapidly increasing coefficients // Izv. AN SSSR. Ser. Matem. 31:5, 1159-1178 (1967). [Math. USSR Izv. 1:5, 1109-1129 (1967).]

9. O.A. Ladyzhenskaya. On the uniqueness of the solution of Cauchy's problem for a linear parabolic equation // Matem. Sborn. 127(69):2, 175-184 (1950). (in Russian).

10. V.F. Gilimshina, F.Kh. Mukminov. On decay rate of solution for degenerating linear parabolic equation // Ufimskij Matem. Zhurn. 3:4, 43-56 (2011). [Ufa Math. J. 3:4, 42-55 (2011).]

11. O.A. Oleinik, E.V. Radkevich. Analyticity and theorems of Liouville and Phragmen-Lindelöf type for general parabolic systems of differential equations // Funkts. Anal. Pril. 8:4, 59-70 (1974). [Funct. Anal. Appl. 8:4, 322-330 (1974).]

12. O.A. Oleinik. The uniqueness of the solution of the Cauchy problem for general parabolic systems in classes of rapidly increasing functions // Uspekhi Matem. Nauk. 29:5, 229-230 (1974). (in Russian.)

13. O.A. Oleinik, G.A. Iosif'yan. An analogue of Saint-Venant's principle and the uniqueness of solutions of boundary value problems for parabolic equations in unbounded domains // Uspekhi Matem. Nauk. [Russ. Math. Surv. 31:4, 153-178 (1976).]

14. L.I. Kamynin, B.N. Khimchenko. Tikhonov-Petrovskii problem for second-order parabolic equations // Sibir. Matem. Zhurn. 22:5, 78-115 (1981). [Sib. Math. J. 22:5, 709-734 (1982).]

15. F.Kh. Mukminov. On uniform stabilization of solutions of the first mixed problem for a parabolic equation // Matem. Sborn. 181:11, 1486-1509 (1990). [Math. USSR Sb. 71:2, 331-353 (1992).]

16. L.M. Kozhevnikova. On uniqueness classes of solutions of the first mixed problem for a quasilinear second-order parabolic system in an unbounded domain // Izv. RAN. Ser. Matem. 65:3, 51-66 (2001). [Izv. Math. 65:3, 469-484 (2001).]

17. V.V. Zhikov. Weighted Sobolev spaces // Matem. Sborn. 189:8, 27-58 (1998). [Sb. Math. 189:8, 1139-1170 (1998).]

18. O.A. Ladyzhenskaya, V.A. Solonnikov, N.N. Ural'tseva. Linear and quasi-linear equations of parabolic type. Nauka, Moscow (1967). [Trans. Math. Mon. 23. Amer. Math. Soc., Providence, RI (1968).]

Venera Fidarisovna Vil'danova,

Bashkir State Pedagogical University named after M. Akhmulla,

October rev. st., 3a,

450000, Ufa, Russia

E-mail: gilvenera@mail.ru

Farit Khamzaevich Mukminov,

Institute of Mathematics CC USC RAS,

Chernyshevskii str., 112,

450008, Ufa, Russia

E-mail: mfkh@rambler.ru 